\title{
An efficient low-cost real-time brain computer interface system based on SSVEP
}

\author{
Ruen Shan Leow ${ }^{1 \mathrm{a})}$, Mahmoud Moghavvemi ${ }^{2 \mathrm{~b}}$, and \\ Fatimah Ibrahim $^{1 \mathrm{c})}$ \\ ${ }^{1}$ Department of Biomedical Engineering, University of Malaya, \\ 50603 Kuala Lumpur, Malaysia \\ ${ }^{2}$ Department of Electrical Engineering, University of Malaya, \\ 50603 Kuala Lumpur, Malaysia \\ a)ruen_shan@yahoo.com \\ b)mahmoud@um.edu.my \\ c)fatimah@um.edu.my
}

Abstract: This paper presents the development of a real-time brain computer interface (BCI) system based on the detection of steady-state visual evoked potential (SSVEP). The system includes a frequencyprogrammable visual stimulator, EEG-band amplifier and filter, 16bit data acquisition card, and signal processing and classification algorithms. Three visual stimuli flickering at varying frequencies were shown to the subject, and the system is able to identify the desired target that the subject was focusing on. Experiments on ten healthy subjects using the designed system yielded an average detection accuracy of $86.15 \%$.

Keywords: Steady-state visual evoked potential (SSVEP), electroencephalography (EEG), brain computer interface (BCI)

Classification: Electronic instrumentation and control

\section{References}

[1] J. R. Wolpaw, et al., "Brain-computer interface technology: a review of the first international meeting," IEEE Trans. Rehabil. Eng., vol. 8, pp. 164173, June 2000.

[2] M. Middendorf, G. McMillan, G. Calhoun, and K. S. Jones, "Braincomputer interfaces based on the steady-state visual-evoked response," IEEE Trans. Neural Syst. Rehabil. Eng., vol. 8, pp. 211-214, June 2000.

[3] E. Lalor, et al., "Brain-Computer Interface based on the steady-state VEP for immersive gaming control," in Proc. 2nd Int. Brain-Computer Interface Workshop and Training Course 2004, pp. 63-64, 2004.

[4] Y. J. Wang, R. P. Wang, X. R. Gao, B. Hong, and S. K. Gao, "A Practical VEP-Based Brain-Computer Interface," IEEE Trans. Neural Syst. Rehabil. Eng., vol. 14, no. 2, pp. 234-239, 2006. 
[5] R. A. Johnson and D. W. Wichern, Applied Multivariate Statistical Analysis, 5th ed., Pearson Education International, pp. 609-640, 2002.

\section{Introduction}

A brain computer interface (BCI) system allows human to communicate with a computer via brain signals, without depending on the brain's normal output pathway of peripheral nerves and muscles [1]. Brain electrical signals can be obtained via invasive or non-invasive methods. Non-invasive method is more favorable in BCI applications due to lower health risk, lower technical cost and convenient approach. Electroencephalography (EEG) is a popular non-invasive method of acquiring electrical potentials from the scalp surface. By acquiring and translating the brain signals into useful commands, BCI system can serve as an alternative method of communication for individuals who have severe neuromuscular problem.

SSVEP is the periodic response elicited in the brain when a person is visually focusing his/her attention on a stimulus that is continuously flickering at frequency $6 \mathrm{~Hz}$ and above [2]. BCI research groups favor SSVEP as input signal due to two reasons. First, SSVEP can be conveniently detected using frequency domain algorithms due to the significant increase in specific power spectrum, and second, SSVEP can be triggered in most subjects without requiring special subject training.

BCI works utilizing SSVEP feature has been reported more often since recent years. One of the pioneers is Middendorf, et al. [2] who developed a SSVEP-based BCI system for a control of flight simulator. Lalor, et al. [3] used SSVEP for binary control of a 3-dimensional computer game. Recently Wang, et al. [4] have designed an environmental controller using multi-class SSVEP-based BCI system. Their system was proved to be applicable to more than $90 \%$ of people with high transfer rate in living environment. These works had demonstrated innovative SSVEP application in BCI systems and successfully addressed low detection accuracy problem in early years.

Current SSVEP research requires complicated and expensive BCI acquisition system. Thus, there is a need to lower the cost using affordable components in order to benefit the general consumer who is interested to develop their own BCI infrastructure. With this goal in mind, it motivates this research to develop a simple, efficient and low cost BCI system that allows rapid detection of SSVEP. The BCI system developed is able to recognize the target stimulus the subject was focusing on when given a choice of three visual stimuli. The system shares common features with previous SSVEP works reported, however the system proposed in this paper is simple and inexpensive, utilizing fewer electronic components. 


\section{Hardware system design}

The system comprises of programmable visual stimulus, EEG-band amplifier with filter, data acquisition card, signal processing and classification algorithms. Figure 1 shows the block diagram of the SSVEP-BCI system, and the schematic of one channel of the amplifier and filter system is shown in Figure 2. The visual stimulus is a $2 \mathrm{~cm}$ diameter red color light emitting diode (LED) with frequency controlled by a PIC16F876 microcontroller chip.

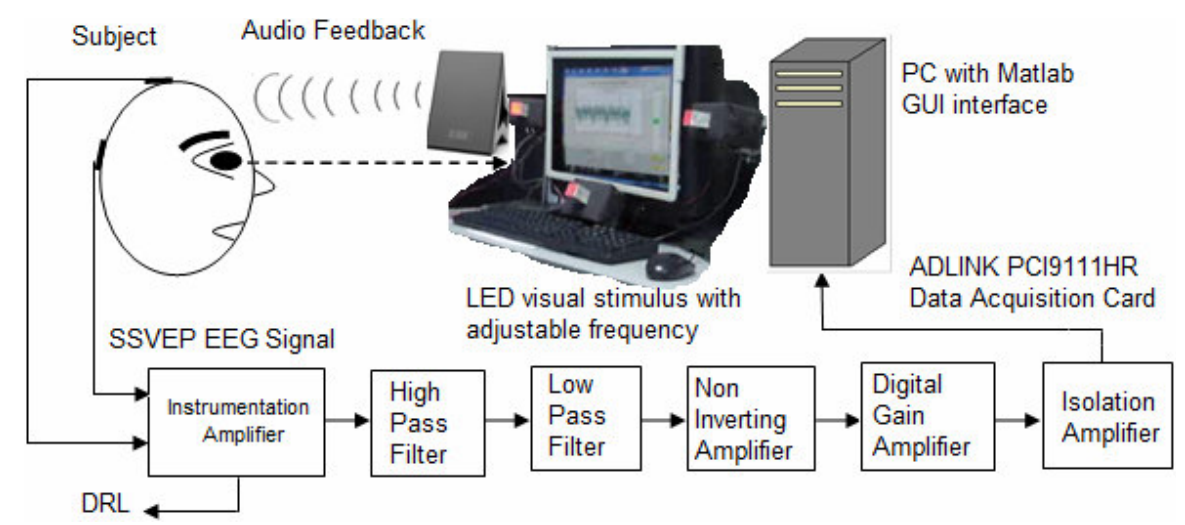

Fig. 1. Block diagram of the SSVEP-BCI system.

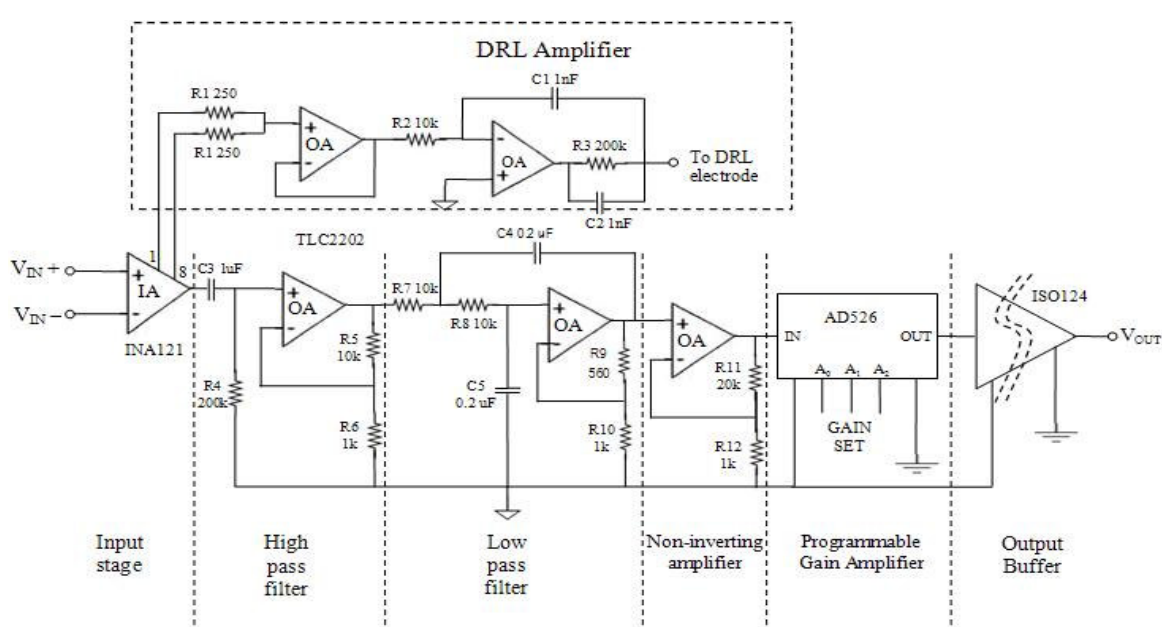

Fig. 2. Schematic of one channel of the amplifier and filter system.

The EEG signal acquisition system is composed of a custom designed two channel differential EEG amplifier which provides an average differential gain of 39342 and a data acquisition card. The first stage of the EEG amplifier is designed using an instrumentation amplifier INA121 to ensure good common mode rejection ratio (CMRR) and high input impedance. The signal is amplified for $101(\sim 40 \mathrm{~dB})$ times at this stage. A high CMRR and moderate gain at initial stage is desirable so that the common mode signals that exist in human body will not be introduced into the resultant output. 
Subsequent high pass and low pass active filters are constructed using op-amps. The lower and upper $-3 \mathrm{~dB}$ frequencies of the first order high pass filter and second order low pass filter are measured as $0.85 \mathrm{~Hz}$ and $70.5 \mathrm{~Hz}$, respectively. After filtering, the signal is amplified by an active non-inverting amplifier for 21 times. A programmable gain amplifier (AD526) and isolation amplifier (ISO124) are included in the latter stage to allow the output gain to be adjusted when necessary, and to isolate the noise. In order to reduce the $50 \mathrm{~Hz}$ interference, a Driven Right Leg (DRL) circuit is used to reduce the common-mode signals.

The entire circuit is constructed on a dual-layer FR-4 printed circuit board (PCB). The CMRR at $50 \mathrm{~Hz}$ is measured as $115.63 \mathrm{~dB}$ and the differential AC input range is approximately $1.8-225 \mu \mathrm{V}$. A dual voltage power supply unit and USB power drawn from a PC USB port are used in parallel to provide bipolar $\pm 5 \mathrm{~V}$ direct current $(\mathrm{DC})$ and unipolar $+5 \mathrm{~V}$ DC respectively, to the amplifier system. Total power consumption of the amplifier system is $67 \mathrm{~mW}$. After being amplified and filtered, the output signal is digitized at sampling rate $1024 \mathrm{~Hz}$ by a 16-bit data acquisition card (ADLINK PCI9111HR) installed in computer.

\section{Signal processing and classification}

The signal processing and classification program are developed using Matlab 6.5.1. Discrete Fourier Transform (DFT) is used to compute the power spectrum of the SSVEP signals at the three stimulus frequencies. One second of EEG data is windowed and zero padded to produce a frequency resolution of $0.5 \mathrm{~Hz}$. Then, the average power spectrum of the two channels is computed. Fisher's Linear Discriminant Analysis (LDA) is used to classify the computed power spectrum and to determine the target frequency with largest power spectrum. The sample pooled covariance matrix is given in equation below [5].

$$
S_{\text {pooled }}=\frac{\left(n_{1}-1\right) S_{1}+\left(n_{2}-1\right) S_{2}}{\left(n_{1}+n_{2}-2\right)}
$$

where

$S_{i}=$ Sample covariance of groups $i, i=1,2$

$n_{i}=$ Number of observations in the groups $i, i=1,2$

To achieve maximum separation of the samples from different groups,

$$
\max _{w} \frac{\left(w^{\prime}\left(\overline{x_{1}}-\overline{x_{2}}\right)\right)^{2}}{w^{\prime} S_{\text {pooled }} w}=\left(\overline{x_{1}}-\overline{x_{2}}\right)^{\prime} S_{\text {pooled }}^{-1}\left(\overline{x_{1}}-\overline{x_{2}}\right)
$$

where

$x_{i}=$ Sample mean of groups $i, i=1,2$

$w=\mathrm{LDA}$ coefficients $=S_{\text {pooled }}^{-1}\left(\overline{x_{1}}-\overline{x_{2}}\right)^{\prime}$

The linear discriminant function $y(x)$ is given below.

$$
y(x)=\log \left(\frac{n_{1}}{n_{2}}\right)-\frac{1}{2}\left(\overline{x_{1}}-\overline{x_{2}}\right)^{\prime} S_{\text {pooled }}^{-1}\left(\overline{x_{1}}+\overline{x_{2}}\right)
$$

For a new observation $x$, it will be allocated into group 1 if $y(x)>0$. Otherwise, $x$ will be allocated into group 2 . 
A graphic user interface is developed using Matlab GUIDE toolbox to provide feedback to the user. At the end of each trial, the detected target will be displayed on the computer screen and audio feedback will be provided.

\section{Experiment procedures}

During the EEG experiment, the subject was seated comfortably on a chair facing the visual stimulus. EEG signals were recorded from positions $\mathrm{O} 1$ and O2 according to the international 10-20 system and referenced to forehead. DRL electrode was placed on the left arm of the subject. Subjects were required to close their eyes and two minutes of REST signals were recorded. After that, subjects were given a few minutes to adapt to the flickering stimulus before the SSVEP sessions started. Subjects were required to participate in preliminary and real-time experiment, carried out on two different days.

Preliminary experiment is conducted to determine three optimal frequencies for each subject. During the preliminary experiment, only one visual stimulus is presented. For each trial, the LED stimulus is programmed to blink for 7 seconds at the selected frequency and dim for 10 seconds. Subjects were required to maintain full visual fixation on the stimulus when it is blinking, and the signals recorded when the subject was focusing at the blinking stimulus is termed SSVEP signals. Frequencies ranging from $7 \mathrm{~Hz}$ to $31 \mathrm{~Hz}$ were tested, and each frequency was tested for at least 5 times.

After determining three optimal frequencies, real-time experiment is carried out. Three LED stimuli placed at the left, bottom and right edge of the computer screen was presented $50 \mathrm{~cm}$ in front of the subject, each programmed to flicker at a particular optimal frequency respectively. SSVEP trials will start when the subject is ready. Subject is required to focus their attention only on one visual stimulus during a trial. At the end of the trial, the system will analyze the SSVEP signals recorded, and the results will be provided.

\section{Results and discussions}

Ten voluntary healthy subjects with normal or corrected-to-normal vision have used the real-time SSVEP-based BCI system to select one of the desired target from three frequency-coded visual stimulus. The classification results are shown in Table I. The results showed that the subjects are more sensitive to frequencies ranging from 20 to $28 \mathrm{~Hz}$, and the highest classification error rate is less than $9.09 \%$.

\section{Conclusion}

In this research, a simple real-time BCI system using minimal numbers of standard off-the-shelf components is proposed. Detection accuracy as high as $86.15 \%$ reported in this work shows that low cost solution for general consumer market is a feasible idea. The system also has the flexibility to be further enhanced. For example the number of selection targets and number of EEG amplifier channels can be increased. Another improvement is 
Table I. Results of ten subjects participated in real-time experiment.

\begin{tabular}{|c|c|c|c|c|}
\hline Subjects & $\begin{array}{c}\text { Optimal } \\
\text { Frequencies (Hz) }\end{array}$ & $\begin{array}{c}\text { Classification } \\
\text { accuracy (\%) }\end{array}$ & $\begin{array}{c}\text { Classification } \\
\text { error }(\%)\end{array}$ & $\begin{array}{c}\text { Percentage of zero } \\
\text { target detected (\%) }\end{array}$ \\
\hline S1 & $14,20.5,23$ & 90.14 & 0 & 9.86 \\
\hline S2 & $21,24,27.5$ & 75.58 & 8.14 & 16.28 \\
\hline S3 & $20.5,23,25.5$ & 100 & 0 & 0 \\
\hline S4 & $24,26,28$ & 72 & 0 & 28 \\
\hline S5 & $24,27.5,29$ & 77.78 & 0 & 22.22 \\
\hline S6 & $20,23,26$ & 85.53 & 2.63 & 11.84 \\
\hline S7 & $22,24,26$ & 93.33 & 5 & 1.67 \\
\hline S8 & $24,26,28$ & 86.67 & 5 & 8.33 \\
\hline S9 & $24,26,28$ & 95.65 & 1.45 & 2.90 \\
\hline S10 & $22,24,26$ & 84.85 & 9.09 & 6.06 \\
\hline Average & - & 86.15 & 3.13 & 10.72 \\
\hline
\end{tabular}

the electrode locations and operating speed for each individual subject can be customized in order to obtain optimized data transfer speed. Lastly the current system can be integrated with external peripherals to allow neuromuscular disabled people utilizing it for specific communication purposes such as computing and home automation. 